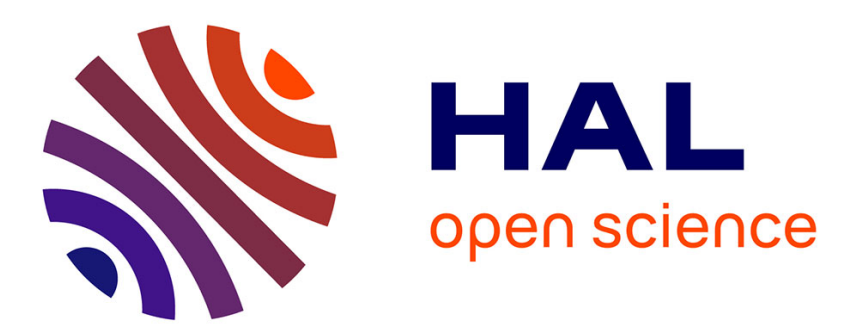

\title{
Optimal Design of Energy-Efficient Cooperative WSNs: How Many Sensors Are Needed?
}

Stefan Mijovic, Luca Sanguinetti, Chiara Buratti, Merouane Debbah

\section{To cite this version:}

Stefan Mijovic, Luca Sanguinetti, Chiara Buratti, Merouane Debbah. Optimal Design of EnergyEfficient Cooperative WSNs: How Many Sensors Are Needed?. 16th IEEE International Workshop on Signal Processing Advances in Wireless Communications, (SPAWC) 2015, Jun 2015, Stockholm, Sweden. 10.1109/SPAWC.2015.7226994 . hal-01242526

\section{HAL Id: hal-01242526 \\ https://hal.science/hal-01242526}

Submitted on 14 Dec 2015

HAL is a multi-disciplinary open access archive for the deposit and dissemination of scientific research documents, whether they are published or not. The documents may come from teaching and research institutions in France or abroad, or from public or private research centers.
L'archive ouverte pluridisciplinaire HAL, est destinée au dépôt et à la diffusion de documents scientifiques de niveau recherche, publiés ou non, émanant des établissements d'enseignement et de recherche français ou étrangers, des laboratoires publics ou privés. 


\title{
Optimal Design of Energy-Efficient Cooperative WSNs: How Many Sensors Are Needed?
}

\author{
Stefan Mijovic ${ }^{* \ddagger}$, Luca Sanguinetti ${ }^{\dagger \ddagger}$, Chiara Buratti* ${ }^{*}$, and Mérouane Debbah ${ }^{\ddagger \S}$ \\ *DEI, University of Bologna, Bologna, Italy \\ ${ }^{\dagger}$ Dipartimento di Ingegneria dell'Informazione, University of Pisa, Pisa, Italy

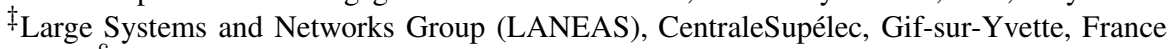 \\ $\S_{\text {Mathematical and Algorithmic Sciences Lab, Huawei France, Paris, France }}$
}

\begin{abstract}
In this paper, a cooperative Multiple-InputMultiple-Output (MIMO) scheme for wireless sensor networks is presented. We consider a network which consists of inexpensive sensor nodes transmitting data towards sinks. We take into account the hardware limitations this kind of devices typically present. Having in mind potentially high number of nodes in the network, we study the asymptotic regime, inspired by recent developments in the massive MIMO field, which simplifies the performance analysis and is shown to be sound even for relatively low number of nodes. The aim is to provide the optimal number of sensor nodes to be deployed and the duration of the channel estimation phase, having in mind energy efficiency as performance metric.
\end{abstract}

\section{INTRODUCTION}

Wireless sensor networks (WSNs) have recently gained increasing attention as practical technology being introduced to different applications. A considerable number of these applications requires transmission of the acquired data over long distances using resources available only at sensor nodes. In this situation, direct transmission from a source node to a sink over fading channel often presents harsh obstacles mainly due to the large amount of energy required to establish a reliable transmission, thus fostering an inefficient use of the batteries.

Multiple-input multiple-output (MIMO) systems are well known for their capability to obtain high spectral efficiency in the presence of fading channels [1] [2]. However, the need to install multiple antennas in sensor nodes can be problematic for economic and practical reasons. To extend the advantages of MIMO systems to devices characterised by a reduced number of antennas, the idea of deploying a cooperative (also known as virtual or distributed) MIMO architecture appears to be very promising. One of the first studies on this topic was presented in [3], where nodes cooperate to establish virtual antenna arrays, which behave as a single multi-antenna terminal. One of the most interesting cooperative MIMO schemes is cooperative beamforming [4]. More recently, in [5] the authors analyse a virtual MIMO (vMIMO) mechanism for energy efficient data gathering in WSNs. Moreover, they propose a distributed heuristic algorithm for vMIMO-aware

L. Sanguinetti is funded by the People Programme (Marie Curie Actions) FP7 PIEF-GA-2012-330731 Dense4Green. This research has also been supported by the FP7 NEWCOM\# (Grant no. 318306) and the ERC Starting MORE (Grant no. 305123). topology construction and routing which enables high energyefficiency.

Despite its promises, the employment of a cooperative MIMO architecture poses several technical challenges mainly because of the large amount of signalling packets required to establish the cooperation among different nodes. In this paper we take a different approach: we devise a simple mechanism which does not require signalling and allows for deployment of cheap sensor nodes with limited hardware capabilities. The scope of this work is to mathematically model a cooperative WSN and obtain the set of design parameters which maximises the energy efficiency of the system. We show the impact of hardware impairments and channel estimation error on the performance of the system. The asymptotic analysis is used to simplify the problem formulation and to find closed form expressions for the optimal pilot length and number of sensors. Numerical simulations show that the obtained results are valid even for a network of relatively small size.

This work is inspired by recent developments in massive MIMO technique for cellular networks [6], [7], [8]. However, in this work we are considering a different scenario with its particular constraints and requirements.

The rest of the paper is organised as follows ${ }^{1}$. Section II describes the system model, Section III presents the optimisation problem we are solving, Section IV contains the analysis of the asymptotic regime. In Section V we present the simulation setup and numerical results and finally, Section VI concludes the paper.

\section{SySTEM AND SIGNAL MODELS}

Consider a WSN composed of $N$ sensors and $K$ sinks, both equipped with a a single antenna, operating over a bandwidth $B$. We assume that the sensors are detecting an event or measuring the same parameter such that the data they are transmitting, $s$, is the same. We consider a block flat-fading channel with $T$ being the coherence time. We assume that the sensors and sinks operate according to a time-division duplex (TDD) protocol shown in Fig. 1 with $T_{s}$ being the time required to transmit a symbol and $M$ being the number

\footnotetext{
${ }^{1}$ The following notation is used. $\mathrm{E}\{\cdot\}$ denotes the expectation while $\mathcal{C N}$ denotes circularly symmetric complex Gaussian random variable. Matrices and vectors are denoted by uppercase boldface and lowercase boldface letters respectively. We use $\otimes$ to denote the Kronecker product, $H$ accounts for the Hermitian operator, $\operatorname{tr}\{\cdot\}$ denotes the trace of matrix.
} 
of symbols within a frame. As seen, the transmission phase is preceded by a training phase in which pilots, of length $\tau$ symbols, are sent by sinks with $0 \leq \tau \leq M$. Pilots enable sensors to estimate the channels. Moreover, pilots are used for synchronisation among nodes such that, during the transmission phase, they start transmitting at the same time. Given the simplicity of the synchronisation scheme, the synchronisation error does not increase with the number of nodes. In the following we assume that nodes are perfectly synchronised. The TDD protocol is assumed to be matched to the coherence time (i.e., $M T_{s} \leq T$ ). Therefore, the channels are considered reciprocal and the sensors can make use of estimates for transmission. Call $h_{n k}$ the channel coefficient from sensor $n$ to sink $k$ and assume that

$$
h_{n k}=\sqrt{d_{n k}} w_{n k}
$$

where $w_{n k} \sim \mathcal{C N}(0,1)$ accounts for the small-scale fading channel and $d_{n k}$ describes the pathloss. We assume that the precoding coefficient used by sensor $n$ is given by

$$
v_{n}=\frac{1}{\sqrt{\lambda_{n}}} \sum_{i=1}^{K} h_{n i}
$$

where $\lambda_{n}=\sum_{i=1}^{K} d_{n i}$ is chosen such that $\mathrm{E}\left\{\left|v_{n}\right|^{2}\right\}=1$. The adopted precoding scheme is fully distributed and rather simple, since nodes need to know only their own composite channel $\sum_{i=1}^{K} h_{n i}$ towards sinks. However, this comes at a cost. The precoding vector does not match the channel towards any of the sinks, instead, it partially matches all of them, which makes it a sub-optimal version of beamforming. In a more compact form, we may write $\mathbf{v}=\left[v_{1}, \ldots, v_{N}\right]^{T}$ as

$$
\mathbf{v}=\sum_{i=1}^{K} \boldsymbol{\Lambda}^{-1 / 2} \mathbf{h}_{i}, \quad \boldsymbol{\Lambda}=\operatorname{diag}\left\{\lambda_{1}, \ldots, \lambda_{N}\right\}
$$

Let $\hat{\mathbf{v}} \in \mathbb{C}^{N \times 1}$ be an estimate of $\mathbf{v}$ and assume that hardware impairments (such as non-linearities in amplifiers, clock drifts, I/Q imbalance in mixers, finite-precision ADCs and so forth) affect transmission. Similar to [9]-[11], we model the hardware impairments as a reduction of the original signals by a factor $\sqrt{\left(1-\epsilon^{2}\right)}$, where $\epsilon$ is the error vector magnitude, and replacing it with Gaussian distortion noise that carries the removed power. Then, the signal received at sink $k$ takes the form [9]-[11]

$$
y_{k}=\mathbf{h}_{k}^{H}\left(\sqrt{p\left(1-\epsilon^{2}\right)} \hat{\mathbf{v}} s+\boldsymbol{\eta}\right)+n_{k}
$$

where $p$ is the transmit power and $n_{k} \sim \mathcal{C N}\left(0, \sigma^{2}\right)$ is the thermal noise and

$$
\boldsymbol{\eta}=\sqrt{p} \epsilon \hat{\mathbf{v}} \otimes \boldsymbol{\xi}
$$

accounts for non-ideal hardware. In the above, we assume $\boldsymbol{\xi} \sim$ $\mathcal{C N}\left(0, \mathbf{I}_{N}\right)$ so that the distortion noise at sensor $n$ is distributed as $\eta_{n} \sim \mathcal{C N}\left(0, p \epsilon^{2}\left|\hat{v}_{n}\right|^{2}\right)$.

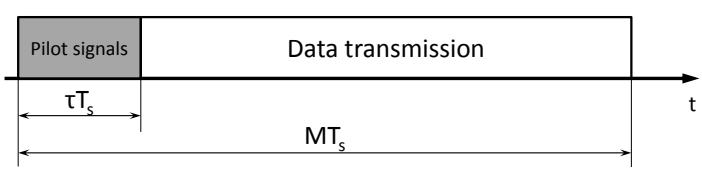

Fig. 1. Time division duplex protocol.

\section{Problem Statement}

The Energy Efficiency (EE) of the WSN is measured in $\mathrm{bit} / \mathrm{Joule}$ and it is computed as the ratio between the achievable rate (in bit/second) and the total power consumption $P_{\mathrm{T}}$ (in Watt $=$ Joule/second). The latter is computed as

$$
P_{\mathrm{T}}=\left(1-\frac{\tau}{M}\right) P_{\mathrm{TX}}+\tau P_{\mathrm{CE}}+N P_{\mathrm{SEN}}+K P_{\mathrm{SINK}}
$$

where $P_{\mathrm{TX}}$ accounts for the power consumption of sensor nodes in transmission state, $P_{\mathrm{CE}}$ is power consumption of sinks in the pilot transmission phase, whereas $P_{\mathrm{SEN}}$ and $P_{\text {SINK }}$ are constant quantities accounting for the fixed power consumption required by each sensor and sink, respectively, for running the circuitry. The objective of this work is to look at the solution of the following problem:

$$
\max _{\{\tau, N\} \in \mathbb{Z}_{+}^{2}} E E=\frac{\left(1-\frac{\tau}{M}\right) \sum_{k=1}^{K} r_{k}}{\left(1-\frac{\tau}{M}\right) P_{\mathrm{TX}}+\tau P_{\mathrm{CE}}+N P_{\mathrm{SEN}}+K P_{\mathrm{SINK}}}
$$

where $r_{k}$ denotes the achievable rate at sink $k$. The pre-log factor $1-\frac{\tau}{M}$ accounts for pilot overhead. In the sequel, we show how to model and compute all the terms in the energy efficiency function in the presence of imperfect channel state information (CSI) and hardware impairments. We start by characterising the statistics of the channel estimation scheme.

\section{A. Pilot-based Estimation of Precoding Coefficients}

Observe that $d_{n i}$ corresponds to the long-term average channel attenuation, which changes in time some orders of magnitudes slower that the fast fading channels. In practice, this means that $d_{n i}$ remains constant for a sufficiently large number of reception phases to be accurately estimated at the sensor. For this reason, in all subsequent discussions we assume that the quantities $\left\{d_{n i}\right\}$ are known at sensor $n$. Therefore, sensor nodes need to estimate only $\sum_{i=1}^{K} h_{n i}$. Assume also that the pilot signal can be represented by a deterministic vector $\mathbf{u} \in \mathbb{C}^{\tau \times 1}$, such that $\left|u_{i}\right|^{2}=p_{\tau}$, with $p_{\tau}$ being a design parameter which defines the transmit power of sinks. Therefore, we have $P_{\mathrm{CE}}=K p_{\tau} / \mu_{\mathrm{SINK}}$, where $\mu_{\text {SINK }} \in(0,1]$ accounts for the transceiver efficiency of sinks.

The collective received signal $\mathbf{x}_{n} \in \mathbb{C}^{\tau \times 1}$ at sensor $n$ is given by

$$
\mathbf{x}_{n}=\sum_{i=1}^{K} h_{n i} \mathbf{u}+\mathbf{n}_{n}=\alpha_{n} \mathbf{u}+\mathbf{n}_{n}
$$

where $\mathbf{n}_{n} \sim \mathcal{C N}\left(0, \varsigma^{2} \mathbf{I}_{N}\right)$ contains the additive noise at the receiver during the pilot signalling. To keep the complexity of 


$$
\begin{aligned}
\gamma_{k} & =\frac{p\left(1-\epsilon^{2}\right)\left|\mathbf{h}_{k}^{H} \boldsymbol{\Lambda}^{-1 / 2} \mathbf{h}_{k}\right|^{2}}{p \frac{\varsigma^{2}}{\tau p_{\tau}} \mathbf{h}_{k}^{H} \boldsymbol{\Lambda}^{-1 / 2} \mathbf{h}_{k}+p\left(1-\epsilon^{2}\right)\left|\sum_{i \neq k} \mathbf{h}_{k}^{H} \boldsymbol{\Lambda}^{-1 / 2} \mathbf{h}_{i}\right|^{2}+p \epsilon^{2} \mathbf{h}_{k}^{H} \mathbf{D h}_{k}+\sigma^{2}} \\
\mathrm{EE} & =\frac{\left(1-\frac{\tau}{M}\right) B \sum_{k=1}^{K} \log _{2}\left(1+\gamma_{k}\right)}{\frac{p}{\mu \operatorname{SEN}}\left(1-\frac{\tau}{M}\right)\left(\mathbf{v}^{H} \mathbf{v}+\frac{\varsigma^{2}}{\tau p_{\tau}} \operatorname{tr}\left\{\boldsymbol{\Lambda}^{-1}\right\}\right)+\frac{\tau p_{\tau}}{\mu \mathrm{SINK}} K+N P_{\mathrm{SEN}}+K P_{\mathrm{SINK}}}
\end{aligned}
$$

sensors at a tolerable level, we employ the least-squares (LS) estimator of $\alpha_{n}$ defined as:

$$
\hat{\alpha}_{n}=\frac{1}{\tau} \mathbf{u}^{H} \mathbf{x}_{n} .
$$

The variance of the estimation error is given by $\mathrm{E}\left\{\mid \alpha_{n}-\right.$ $\left.\left.\hat{\alpha}_{n}\right|^{2}\right\}=\frac{\varsigma^{2}}{\tau p_{\tau}}$. Plugging (10) into (11) and using (2) yields

$$
\hat{\mathbf{v}}=\mathbf{v}+\mathbf{e}
$$

where $\mathbf{e} \in \mathbb{C}^{N \times 1}$ is the estimation error vector with elements $e_{n} \sim \mathcal{C N}\left(0, \frac{1}{\lambda_{n}} \frac{\varsigma^{2}}{\tau p_{\tau}}\right)$.

Observe that a single pilot signal (i.e., $\tau=1$ ) from all sinks would be sufficient to estimate the precoding coefficients at all sensors. This is a consequence of the adoption of precoding scheme in (2). Different precoding schemes based on knowledge of $\left\{h_{n i}\right\}$ would require $\tau \geq K$.

\section{B. Energy Efficiency}

Plugging (12) into (4) and using (3) one gets

$$
\begin{aligned}
y_{k} & =\sqrt{p\left(1-\epsilon^{2}\right)} \mathbf{h}_{k}^{H} \boldsymbol{\Lambda}^{-1 / 2} \mathbf{h}_{k} s+\sqrt{p\left(1-\epsilon^{2}\right)} \mathbf{h}_{k}^{H} \mathbf{e} s+ \\
& +\sqrt{p\left(1-\epsilon^{2}\right)} \sum_{i=1, i \neq k}^{K} \mathbf{h}_{k}^{H} \boldsymbol{\Lambda}^{-1 / 2} \mathbf{h}_{i} s+\epsilon \sqrt{p} \mathbf{h}_{k}^{H} \boldsymbol{\eta}+n_{k} .
\end{aligned}
$$

The achievable rate at sink $k$ is [10], [11]

$$
r_{k}=B \log _{2}\left(1+\gamma_{k}\right)
$$

where $\gamma_{k}$ is the signal-to-interference-plus-noise ratio (SINR) given by (6), shown at the top of page, with $\mathbf{D}$ being defined as $\mathbf{D}=\operatorname{diag}\left\{\left|v_{1}\right|^{2}, \ldots,\left|v_{N}\right|^{2}\right\}$. From (4), it turns out that

$$
P_{\mathrm{TX}}=\frac{1}{\mu_{\mathrm{SEN}}}\left[p\left(1-\epsilon^{2}\right) \mathrm{E}\left\{\hat{\mathbf{v}}^{H} \hat{\mathbf{v}}\right\}+p \epsilon^{2} \mathrm{E}\left\{\boldsymbol{\eta}^{H} \boldsymbol{\eta}\right\}\right]
$$

where $\mu_{\mathrm{SEN}} \in(0,1]$ accounts for the transceiver efficiency of sensor nodes. Using simple calculus we obtain

$$
P_{\mathrm{TX}}=\frac{p}{\mu_{\mathrm{SEN}}}\left(\mathbf{v}^{H} \mathbf{v}+\frac{\varsigma^{2}}{\tau p_{\tau}} \operatorname{tr}\left\{\boldsymbol{\Lambda}^{-1}\right\}\right) .
$$

Putting all the above results together, we have that the EE takes the form in (7), shown at the top of page.

\section{Asymptotic ANALYsis}

To gain some insights into the structure of the solution to (9), we assume that the number of sensors $N$ grows without bound while the number of sinks is kept fixed. For notational convenience, we denote

$$
\mathcal{A}_{k}=\mathrm{E}\left\{\frac{d_{k}}{\sqrt{\sum_{i=1}^{K} d_{i}}}\right\} \quad \mathcal{B}_{k}=\mathrm{E}\left\{\frac{d_{k}}{\sum_{i=1}^{K} d_{i}}\right\}
$$

and

$$
\mathcal{C}_{k}=\mathrm{E}\left\{\frac{d_{k}^{2}}{\sum_{i=1}^{K} d_{i}}\right\} \quad \mathcal{D}_{k}=\sum_{\ell \neq k} \mathrm{E}\left\{\frac{d_{k} d_{\ell}}{\sum_{i=1}^{K} d_{i}}\right\} .
$$

Observe that the above coefficients depend only on the average channel attenuations. The following result can be easily proved.

Lemma 1. If $N$ grows without bound, then $\frac{1}{N} \gamma_{k}-\bar{\gamma}_{k} \rightarrow 0$ almost surely with

$$
\bar{\gamma}_{k}=\frac{p\left(1-\epsilon^{2}\right)\left|\mathcal{A}_{k}\right|^{2}}{p \frac{\varsigma^{2}}{\tau p_{\tau}} \mathcal{B}_{k}+p \epsilon^{2}\left(2 \mathcal{C}_{k}+\mathcal{D}_{k}\right)} .
$$

Moreover, we have that $\frac{1}{N} P_{\mathrm{TX}}-\bar{P}_{\mathrm{TX}} \rightarrow 0$ almost surely with

$$
\bar{P}_{\mathrm{TX}}=\frac{p}{\mu_{\mathrm{SEN}}}\left(1+\frac{\varsigma^{2}}{\tau p_{\tau}} \mathrm{E}\left\{\frac{1}{\sum_{i=1}^{K} d_{i}}\right\}\right) .
$$

Proof: The results easily follow using simple statistical arguments and from observing that $\mathbf{v}^{H} \mathbf{v}=$ $\sum_{i=1}^{K} \mathbf{h}_{i}^{H} \boldsymbol{\Lambda}^{-1} \mathbf{h}_{i}$ from which using (1) and (3) it follows that $\frac{1}{N} \sum_{i=1}^{K} \mathbf{h}_{i}^{H} \boldsymbol{\Lambda}^{-1} \mathbf{h}_{i}-1 \rightarrow 0$ almost surely.

Lemma 1 shows that the SINR and the transmit power increase linearly with $N$. Although valid for $N$ growing without bounds, next we use this result for a system with a large but finite number of sensors. Using (19) and (20) into (7) leads to

$$
\overline{\mathrm{EE}}=\frac{\left(1-\frac{\tau}{M}\right) B \sum_{k=1}^{K} \log _{2}\left(1+\bar{\gamma}_{k} N\right)}{\left(1-\frac{\tau}{M}\right) \alpha(\tau) N+\beta \tau+N P_{\mathrm{SEN}}+K P_{\mathrm{SINK}}}
$$

where we have defined (for notational compactness)

$$
\alpha(\tau)=\frac{p}{\mu_{\mathrm{SEN}}}\left(1+\frac{\varsigma^{2}}{\tau p_{\tau}} \mathrm{E}\left\{\frac{1}{\sum_{i=1}^{K} d_{i}}\right\}\right)
$$

and $\beta=K p_{\tau} / \mu_{\text {SINK }}$. 
TABLE I

Simulation PARAMETERS

\begin{tabular}{|c|c|c|c|}
\hline Parameter & Value & Parameter & Value \\
\hline Area side length: $a$ & $500 \mathrm{~m}$ & Transceiver efficiency sensor nodes: $\mu_{\mathrm{SEN}}$ & 0.08 \\
\hline Path-loss model: $d_{n k}$ & $10^{-3.53}$ distance $_{n k}^{-3.76}$ & Transceiver efficiency sinks: $\mu_{\text {SINK }}$ & 0.3 \\
\hline Sensor nodes transmit power: $p$ & $1 \mathrm{~mW}$ & Hardware impairments: $\epsilon^{2}$ & 0.17 \\
\hline Pilot transmit power: $p_{\tau}$ & $100 \mathrm{~mW}$ & Bandwidth: $B$ & $5 \mathrm{MHz}$ \\
\hline Circuit power sensor nodes: $P_{\mathrm{SEN}}$ & $20 \mathrm{~mW}$ & Frame duration: $T$ & $8.2 \mathrm{~ms}$ \\
\hline Circuit power sinks: $P_{\text {SINK }}$ & $100 \mathrm{~mW}$ & Number of symbols in a frame: $M$ & 256 \\
\hline Total noise power: $B \sigma^{2}$ & $-107 \mathrm{dBm}$ & Symbol time: $T_{S}$ & $32 \mu \mathrm{s}$ \\
\hline
\end{tabular}

Lemma 2. For $N$ and $K$ given, the value of $\tau$ maximising (21) is

$$
\tau^{\star}=\left\lceil\frac{1}{M} \frac{1 \pm \sqrt{\frac{M^{2}}{c N}\left(\frac{N P_{\mathrm{SEN}}+K P_{\mathrm{SINK}}}{M}+\beta\right)}}{1-\frac{M^{2}}{c N}\left(\frac{N P_{\mathrm{SEN}}+K P_{\mathrm{SINK}}}{M}+\beta\right)}\right\rceil
$$

with

$$
c=\frac{p}{\mu_{\mathrm{SEN}}} \frac{\varsigma^{2}}{p_{\tau}} \mathrm{E}\left\{\frac{1}{\sum_{i=1}^{K} d_{i}}\right\} .
$$

Proof: The result follows setting $\partial \overline{\mathrm{EE}} / \partial \tau=0$ and solving with respect to $\tau$.

Finding the optimal $N^{\star}$ for a given $\tau$ is very hard due to the sum over the number of sinks. A possible setting in which this can be easily accomplished is when the sinks are uniformly distributed over a circle. In these circumstances, the symmetry implies that $\bar{\gamma}_{k}=\bar{\gamma}, \forall k$ so that (21) reduces to

$$
\overline{\mathrm{EE}}=\frac{\left(1-\frac{\tau}{M}\right) B K \log _{2}(1+\bar{\gamma} N)}{\alpha N+\beta \tau+K P_{\mathrm{SINK}}} .
$$

We now look at the EE-optimal value of $N$ when $\tau$ is given.

Lemma 3. For $\tau$ given, the value of $N$ maximising (25) is given by

$$
N^{\star}=\frac{e^{\left(z^{\star}+1\right)}-1}{\bar{\gamma}}
$$

where

$$
z^{\star}=W\left(\frac{\bar{\gamma}\left(\beta \tau+K P_{\mathrm{SINK}}\right)}{\alpha e}-\frac{1}{e}\right)
$$

and $W(x)$ is the Lambert function defined by the equation $t=W(t) e^{W(t)}$ for any $t \in \mathbb{C}$.

Proof: The proof relies on using the same arguments of Theorem 2 in [7] and is omitted for space limitations.

\section{NUMERICAL RESUlTS}

The above presented model has been implemented in Matlab. Sensor nodes and sinks are assumed to be uniformly distributed within the observation area, a square of side $a$. In order to get stable results, we average over 1000 realisations of positions and all the other random variables, such as noise, fading, etc. The network parameters are given in Table I. Those related to the energy consumption are taken from the datasheet of a popular WSN device, TI CC2530 [12], while PHY layer parameters are inspired by the IEEE 802.15.4 standard [13].

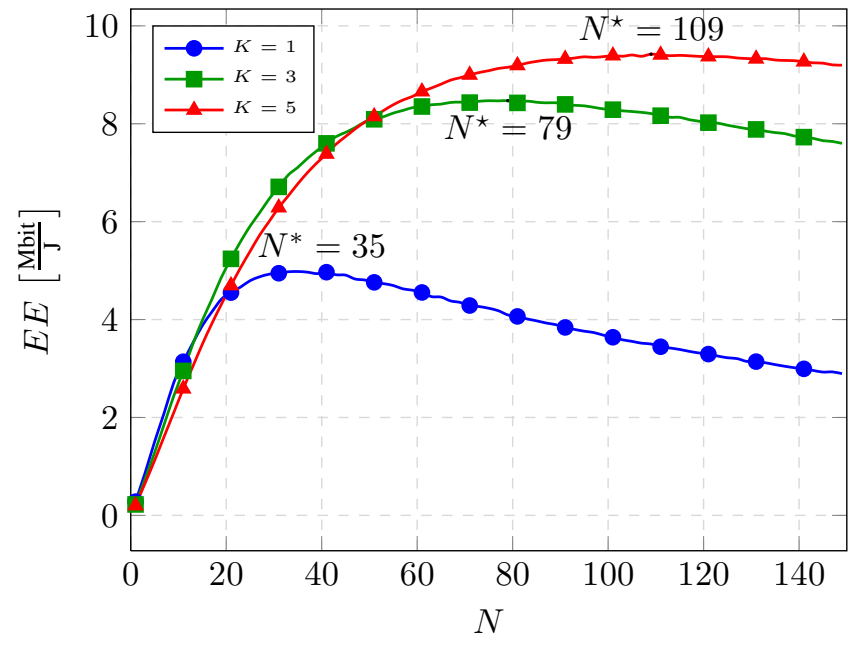

Fig. 2. Energy efficiency as a function of $N$ for $\tau=5$.

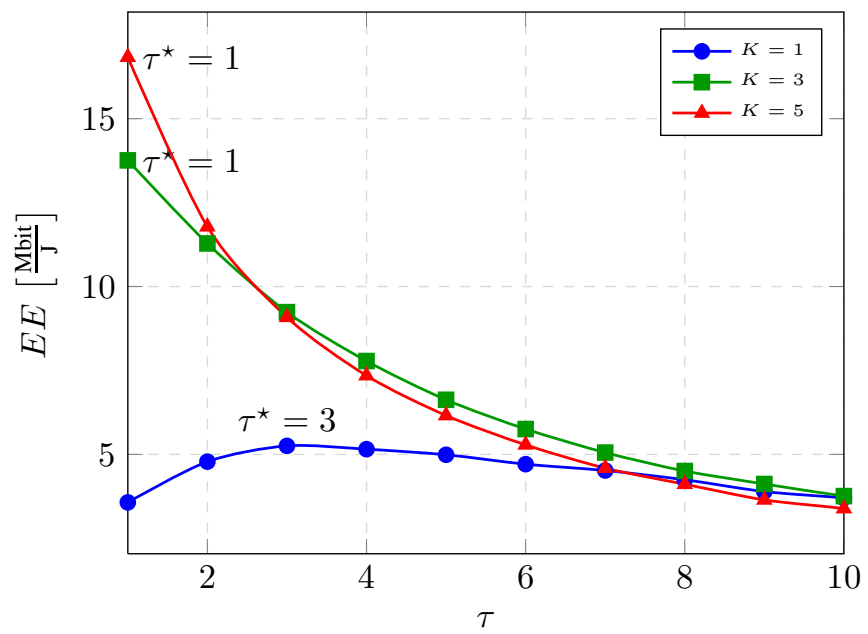

Fig. 3. Energy efficiency as a function of $\tau$ for $N=30$.

Fig. 2 illustrates the energy efficiency as a function of $N$ for different values of $K$ when $\tau=5$. As can be seen, increasing $K$ improves energy efficiency but the optimal number of nodes $N$ needs to be increased accordingly. Observe that the maximum region of the curve tends to become flat as $K$ becomes large, meaning that we can admit certain error in the computation of the optimal $N$ without losing in performance. This will be useful later on.

Fig. 3 illustrates the energy efficiency as a function of $\tau$ when $N=30$ and $K=1,3$ and 5 . As it can be observed, the EE-optimal value of $\tau$ decreases when increasing 


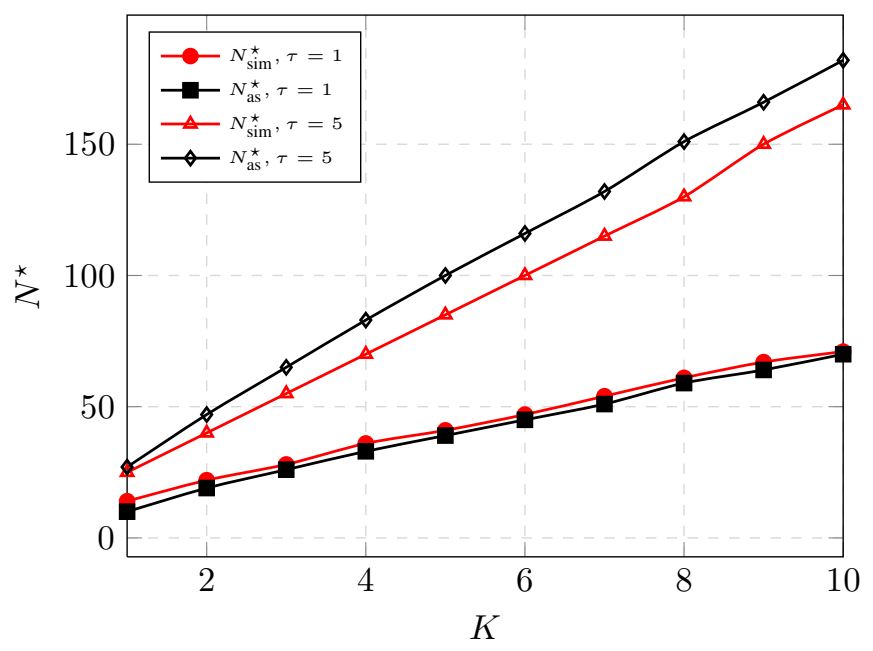

Fig. 4. Optimal number of sensor nodes, $N^{\star}$ as a function of $K$.

$K$. This is due to the fact that the energy consumption of pilot transmission phase increases with $K$ and becomes the dominant part of the overall energy consumption, mostly because pilots are transmitted with high power. In this setting, the gain in terms of achievable rate obtained by increasing $\tau$ is counterweighted by the increase in energy consumption.

The results of Figs. 4 and 5 refer to the asymptotic analysis. In particular, Fig. 4 shows the optimal number of sensor nodes, $N^{\star}$, as a function of $K$ for $\tau=1$ and 5 . We can see that $N^{\star}$ increases linearly with $K$ with a slope that depends on $\tau$. The difference between the values of $N^{\star}$ obtained by simulations, $N_{\text {sim }}^{\star}$, and asymptotic analysis, $N_{\text {as }}^{\star}$, does not exceed $10 \%$. Fig. 5 illustrates the energy efficiency achieved by the optimal number of sensor nodes $N_{\text {sim }}^{\star}$ and $N_{\text {as }}^{\star}$ as a function of $K$ for different $\tau$. As it is seen, $N_{\text {as }}^{\star}$ achieves the same performance as $N_{\text {sim }}^{\star}$, meaning that it is safe to use (26), instead of running simulations, in order to get the EE-optimal value of $N$.

\section{CONCLUSIONS}

In this paper we presented a cooperative MIMO scheme for WSNs. The presented scheme is simple and introduces very little overhead. We analyse the performance in terms of energy efficiency and optimise the number of sensor nodes as well as the duration of channel estimation phase. The EE-optimal number of sensor nodes increases linearly with the number of sinks. The analysis shows that as the number of sinks increases, the energy consumption due to pilots transmission becomes dominant and, consequently, the EE-optimal duration of the channel estimation phase gets shorter. We also show that when all the sinks are symmetrically distributed within the observation area, the optimisation problem is simplified and asymptotic analysis provides a closed form solution for the optimal number of sensor nodes.

\section{REFERENCES}

[1] G. J. Foschini, "Layered space-time architecture for wireless communication in a fading environment when using multi-element antennas," Bell Labs Technical Journal, vol. 1, no. 2, pp. 41-59, Autumn 1996.

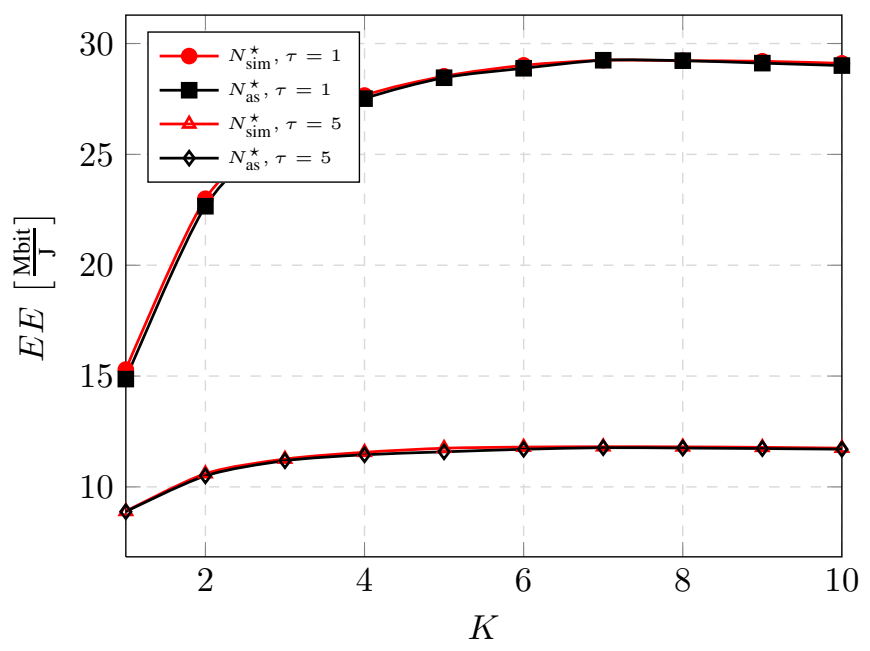

Fig. 5. Energy efficiency achieved by $N_{\text {sim }}^{\star}$ and $N_{\text {as }}^{\star}$, as a function of $K$.

[2] E. Telatar, "Capacity of Multi-antenna Gaussian Channels," European Transactions on Telecommunications, vol. 10, no. 2, p. 585595, Autumn 1999.

[3] M. Dohler, A. Gkelias, and H. Aghvami, "A resource allocation strategy for distributed MIMO multi-hop communication systems," IEEE Communications Letters, vol. 8, no. 2, pp. 99-101, Feb 2004.

[4] G. Barriac, R. Mudumbai, and U. Madhow, "Distributed beamforming for information transfer in sensor networks," in Third International Symposium on Information Processing in Sensor Networks, April 2004, pp. 81-88.

[5] H. Xu, L. Huang, C. Qiao, W. Dai, and Y. e Sun, "Joint Virtual MIMO and Data Gathering for Wireless Sensor Networks," IEEE Transactions on Parallel and Distributed Systems, vol. 26, no. 4, pp. 1034-1048, April 2015.

[6] J. Hoydis, S. ten Brink, and M. Debbah, "Massive MIMO in the UL/DL of Cellular Networks: How Many Antennas Do We Need?" IEEE Journal on Selected Areas in Communications, vol. 31, no. 2, pp. 160-171, February 2013.

[7] E. Bjornson, L. Sanguinetti, J. Hoydis, and M. Debbah, "Optimal Design of Energy-Efficient Multi-User MIMO Systems: Is Massive MIMO the Answer?" IEEE Trans. Wireless Commun., To appear, 2015.

[8] E. Björnson, L. Sanguinetti, and M. Kountouris, "Deploying dense networks for maximal energy efficiency: Small cells meet massive MIMO," IEEE J. Sel. Areas Commun., submitted, http://arxiv.org/abs/1505.01181.

[9] W. Zhang, "A General Framework for Transmission with Transceiver Distortion and Some Applications," IEEE Trans. Commun., vol. 60, no. 2, pp. 384-399, Feb. 2012.

[10] E. Bjornson, P. Zetterberg, M. Bengtsson, and B. Ottersten, "Capacity Limits and Multiplexing Gains of MIMO Channels with Transceiver Impairments," IEEE Commun. Lett., vol. 17, no. 1, pp. 91-94, Jan. 2013.

[11] E. Björnson, J. Hoydis, M. Kountouris, and M. Debbah, "Massive MIMO Systems With Non-Ideal Hardware: Energy Efficiency, Estimation, and Capacity Limits," IEEE Trans. Inf. Theory, vol. 60, no. 11, pp. 7112 - 7139, Nov 2014.

[12] "Texas Instruments CC2530 - Second Generation System-on-Chip Solution for 2.4 GHz IEEE 802.15.4 / RF4CE / ZigBee ," http://www.ti. com/product/cc2530.

[13] "IEEE Standard for Local and metropolitan area networks-Part 15.4: Low-Rate Wireless Personal Area Networks (LR-WPANs)," http:/l standards.ieee.org/getieee802/download/802.15.4-2011.pdf, 2011. 